\title{
Optical Engineering
}

\section{Infrared acousto-optic tunable filter point spectrometer for detection of organics on mineral surfaces}

Rula Tawalbeh

David Voelz

David Glenar

Xifeng Xiao

Nancy Chanover

Robert Hull

David Kuehn 


\section{Infrared acousto-optic tunable filter point spectrometer for detection of organics on mineral surfaces}

\author{
Rula Tawalbeh \\ David Voelz \\ New Mexico State University \\ Las Cruces, New Mexico 88003-0001 \\ E-mail: davvoelz@nmsu.edu
}

\section{David Glenar}

University of Maryland

Baltimore County

1000 Hilltop Circle

Baltimore, Maryland 21250

\section{Xifeng Xiao \\ Nancy Chanover}

Robert Hull

New Mexico State University

Las Cruces, New Mexico 88003-0001

\section{David Kuehn}

Pittsburg State University

1701 South Broadway

Pittsburg, Kansas 66762

\begin{abstract}
A prototype infrared (IR) acousto-optic tunable filter (AOTF)based point spectrometer has been designed for examining and analyzing potential biological samples collected in situ from the planets or other solar system objects. The reflectance spectrometer operates at a wavelength range of 1.6 to $3.6 \mu \mathrm{m}$, which is diagnostic of minerals and organics, and inspects a 1-mm sized spot on the sample. The tuning component is the AOTF that has been utilized in a variety of spectral detection applications. The instrument's specification and design approach including the selected components is described. The data acquisition system, the electronic components, and their interconnections are presented. The instrument's radiometric performance is examined and described by a noise equivalent reflectance value of $0.13 \%$ that is obtained from the laboratory measurements. The device has been demonstrated by measuring the reflectance spectra for a variety of geological samples and comparing the results with the United States Geological Survey data. (๑ The Authors. Published by SPIE under a Creative Commons Attribution 3.0 Unported License. Distribution or reproduction of this work in whole or in part requires full attribution of the original publication, including its DOI. [DOI: 10.1117/1.OE.52.6.063604]
\end{abstract}

Subject terms: acousto-optic tunable filter; spectrometer; radiometry; spectral resolution; NASA space and planetary exploration; United States Geological Survey.

Paper 130245 received Feb. 19, 2013; revised manuscript received May 10, 2013; accepted for publication May 29, 2013; published online Jun. 21, 2013.

\section{Introduction}

Infrared (IR) reflectance spectroscopy is a powerful diagnostic tool for analyzing the composition of a reflecting surface. Absorption features in near-infrared (NIR) spectra $<4 \mu \mathrm{m}$ can be associated with the molecular functional groups and provide information about the molecular structure of a sample. IR spectroscopy is applied in chemical and physical analyses, remote sensing, astronomy, and various industrial applications.

New Mexico State University (NMSU) teamed with NASA/Goddard Space Flight Center (GSFC) to develop a joint instrument comprised of an IR reflectance pointspectrometer (PS) and a laser desorption time-of-flight mass spectrometer (LDTOF-MS). The purpose of the instrument is to examine and analyze samples collected in situ from the planetary or other solar system surfaces and search for organic biomarkers or mineralogical signatures that imply the existence or lack of organic materials. ${ }^{1}$

We include here a short, selective review of the acoustooptic tunable filter (AOTF) applications in passive remote sensing. AOTFs have been employed for a variety of applications including microscopy, spectroscopy, and hyperspectral imaging. For example, in Ref. 2, a fluorescence AOTF microscope is described that provides a fast spectra collection with flexible operational options. In this spectrometer, the AOTF replaces conventional fluorescence excitation and detection filters to provide superior filter switching speed with continuous tunability throughout the visible spectrum. The AOTF has also been used for cytometry applications as a part of a multispectral imaging system, ${ }^{3}$ where the AOTF was used instead of a liquid crystal tunable filter to help reduce system cost and complexity. In image cytometry, high spectral resolution is not required so that an affordable AOTF with modest spectral resolution can be used.

Because of their small size and simplicity of operation, AOTFs have a heritage in astronomical applications. In Ref. 4, a compact, in situ, two-channel AOTF tunable camera was developed at GSFC as a lander mission demonstration instrument under the Mars Instrument Development Program. Two AOTFs were used to provide two spectral channels; one covering the visible/near infrared wavelength range from 0.5 to $1.02 \mu \mathrm{m}$ and another covering the short wave infrared range from 0.8 to $2.4 \mu \mathrm{m}$. In Ref. 5, AOTFs were used in the spectroscopy for the investigation of the characteristics of the atmosphere of Venus (SPICAV) instrument aboard the Venus Express orbiter that began operation around Venus in 2006. One AOTF is used for a visible/IR channel $(0.25$ to $1.7 \mu \mathrm{m})$ and another is used for solar occultation measurements $(\sim 2.5$ to $4.0 \mu \mathrm{m})$. Imaging at multiple wavelengths allows investigators to study the three-dimensional structure and dynamics of Venus' atmosphere, including cloud layers and chemical composition.

An AOTF was also used in the NIR instrument as a part of the spectroscopy for the investigation of the characteristics of the atmosphere of Mars (SPICAM) light optical package for the European Space Agency Mars Express mission. The wavelength range covered is 1 to $1.7 \mu \mathrm{m}$, where the SPICAM was used for $\mathrm{CO}_{2}$ and $\mathrm{H}_{2} \mathrm{O}$ detection. ${ }^{6}$ Korablev et al. ${ }^{7}$ describe the use of the SPICAM measurement to study solar occultation, limb and nadir in the NIR and UV spectral ranges.

An imaging instrument for ground-based astronomy was introduced in Ref. 8, where an AOTF provides polarization and spectral sensitive imaging. Three noncollinear filters 
functioning over the spectral range of 0.5 to $3.5 \mu \mathrm{m}$ were characterized in Ref. 9. Two of the filters were used for imaging applications over different wavelength ranges. The third filter was evaluated over a range of 1.7 to $3.4 \mu \mathrm{m}$ and the effects of diffracted wavelength, incident angle, and bandpass width as a function of radio frequency (RF) were investigated. Acousto-optic imaging was also used in Ref. 10 where images of Titan were collected. A chargecoupled device (CCD) camera system was coupled with the AOTF, which is operated over a wavelength range of 0.5 to $1.05 \mu \mathrm{m}$. In Ref. 11, multispectral imagery of Jupiter and Saturn were obtained with an AOTF camera working over a wavelength range of 0.7 to $0.95 \mu \mathrm{m}$. An example of AOTF-based spectroscopy is the configurablebandwidth imaging spectrometer based on an AOTF that was presented in Ref. 12. This is an autonomous tunable filtering system, where the AOTF was used to select different spectral wavelengths in both the visible and NIR range for sequential imaging. In this case, the RF signal input to the AOTF was generated by a direct digital synthesizer (DDS) that optimizes the RF power for each wavelength. The wavelength range covered was 0.45 to $0.8 \mu \mathrm{m}$. Another example of an imaging instrument is described in Ref. 13, where the AOTF was combined with a liquid-crystal variable retardation plate, a CCD camera and the required optics to allow spectropolarimetric image collection. The wavelength range for this device is 0.4 to $0.8 \mu \mathrm{m}$. Two retardation values associated with vertical and horizontal polarizations are used to acquire each spectral image. Radiation emitted by thermally excited samples in the wavelength range of 1.5 to $3 \mu \mathrm{m}$ was detected by an NIR emission spectrometer based on an AOTF in Ref. 14.

AOTFs are compact, rugged, efficient, portable, space qualified, and effective at low temperatures. An AOTF (noncollinear) is constructed from a birefringent crystal, typically tellurium dioxide $\left(\mathrm{TeO}_{2}\right)$. Broadband unpolarized light enters the crystal where the birefringence initially separates the paths of the two orthogonal linear polarizations (Fig. 1). These two components are identified as the ordinary $(o)$ and extraordinary $(e)$ beams. An RF signal, coupled into the crystal through a piezoelectric transducer, creates an acoustic wave that transits the crystal. The interaction of the light and acoustic wave produces narrowband components with polarizations that are orthogonal to the $o$ and $e$ beams. The narrowband components, indicated by $o^{\prime}$ and $e^{\prime}$, travel with the $o$ and $e$ beams within the crystal. However, at the crystal exit face, phase matching conditions force the narrowband beams to travel at an angle to the $o$ and $e$ beams (see Fig. 1). It is the angular separation of the narrowband

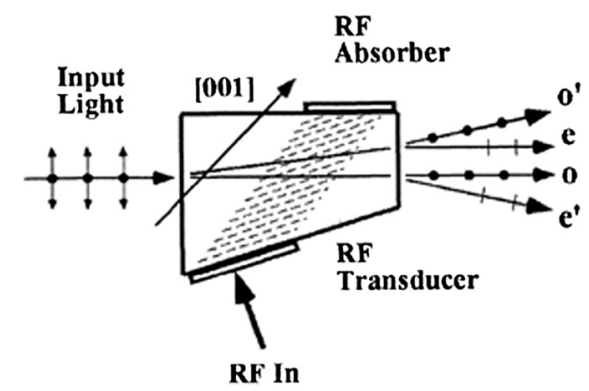

Fig. 1 AOTF schematic diagram indicating the various output beams (Ref. 15). light that allows the device to act as a spectral filter. Furthermore, the center optical wavelengths of the two narrowband components are a function of the input RF, so the device acts as an electronically tunable optical filter.

The center wavelength of the AOTF passband can be tuned quickly by changing the RF. Therefore, its response time can be much shorter than the time required for mechanically changing filters. The AOTF can also be used in a scan mode to function as a spectrometer. With no moving parts, the AOTF is less susceptible to mechanical failure and its performance is not affected by the input intensity and only slightly affected by temperature change.

This paper describes the design, implementation, and testing of the prototype PS developed for the joint instrument (mentioned at the beginning of this section). The PS is based on AOTF technology, which provides some unique advantages over conventional spectrometer approaches in terms of operational flexibility. Based on preliminary mission parameters, the PS is designed to illuminate and examine a $1-\mathrm{mm}$ diameter spot on a sample surface from a few millimeters distance. A reflectance spectrum is measured in the wavelength range of 1.6 to $3.6 \mu \mathrm{m}$ with a spectral resolution width ranging from $4 \mathrm{~nm}$ (FWHM) at the shortest wavelength to about $12 \mathrm{~nm}$ at the longest wavelength ( $R=\lambda / \Delta \lambda$ ranging from 400 to 200 ).

Our PS, while inspired by the instrumentation described above, is fundamentally different from the remote sensing or imaging systems in that it is a nonimaging, active illumination (provides its own light source) device that operates close to the surface of interest. In effect, the AOTF is used to create a tunable narrowband illumination source rather than acting as a filter at the detector. The use of the AOTF for the PS instrument facilitates the joint combination of a reflectance spectrometer with the LDTOF-MS where these two instruments observe the same sample point. The AOTF also simplifies the hardware in that only a single element detector is required, rather than an array that might be used with a conventional grating spectrometer. Furthermore, the AOTF provides operational flexibility in terms of the selection of wavelength scan ranges and observation dwell time.

The PS went through several different design stages before arriving at the current configuration reported here. The resulting arrangement minimizes the overall size and the number of elements in the coupling optics. In this paper, we emphasize the PS design, configuration, and performance. Details of the PS/LDTOF-MS pairing arrangement, mission comments, and initial PS measurements made with breadboard prototypes can be found in the previous publications. ${ }^{1,16}$

\section{Instrument Specifications and Design Approach}

The operational concept for the PS is as follows. Light from a small thermal IR lamp is sent through the AOTF and the RF drive signal is such that the center wavelength of the resulting narrowband light is scanned in time over the range of interest. A mirror directs the filtered light to the sample where a small spot is formed. Light scattered from the sample is collected by a mirror arrangement and is focused on a single element IR detector. Electronics synchronize the scanning of the RF drive signal and the digitization of the detector signal. Some of the more interesting design features of the instrument were developed as a result of the compact size 
Table 1 PS components.

\begin{tabular}{ll}
\hline \hline Component & \multicolumn{1}{c}{ Specifications } \\
\hline IR lamp & $\begin{array}{l}\text { Filament size } 0.6 \times 2 \mathrm{~mm}^{2} ; \text { Required } \\
\text { power } 2.4 \mathrm{~W} \text {; Radiant temperature } 1900 \mathrm{~K}\end{array}$ \\
ZnSe lenses & $\begin{array}{l}\text { Diameter } 12.7 \mathrm{~mm} \text {; Focal length } 12.7 \mathrm{~mm} ; \\
\text { Transmission range } 0.6 \text { to } 6 \mu \mathrm{m}\end{array}$ \\
AOTF & $\begin{array}{l}\text { Crystal } \mathrm{TeO}_{2} ; \text { Wavelength range } 1.7 \text { to } 3.4 \mu \mathrm{m} ; \\
\lambda / \Delta \lambda=200-400\end{array}$ \\
OAP mirrors & $\begin{array}{l}\text { Diameter } 25.4 \mathrm{~mm} \text {; Effective focal length } 25.4 \mathrm{~mm} ; \\
f \text {-number }=1 ; \text { Reflection angle } 90 \text { deg }\end{array}$ \\
\hline \hline
\end{tabular}

requirement and the need to couple the instrument with the LDTOF-MS (Table 1).

The source, optics, and detector arrangement for the final PS design are shown schematically in Fig. 2. In the following sections, we discuss the rationale that led to the design and component choices.

\subsection{Infrared Source}

The source is a compact IR lamp in a TO- 8 package with a can diameter of about $12.7 \mathrm{~mm}$. The lamp's filament is a coil whose projected area transverse to the optical axis is about $2 \times 0.6 \mathrm{~mm}^{2}$ although much of the exitance appears to come from a center 1-mm portion of the filament. A spherical reflector, usually placed behind the filament by the manufacturer to help produce a more directed output beam, is removed as it tends to increase scattered light in our arrangement. The lamp requires about $2.2 \mathrm{~V}$ and $1.1 \mathrm{~A}$ of current. The source's effective temperature is about $1900 \mathrm{~K}$ although the emissivity of the tungsten filament is expected to be only $10 \%$ to $20 \%$ at NIR wavelengths. ${ }^{17}$

\subsection{Zinc Selenide Lenses}

Three identical zinc selenide ( $\mathrm{ZnSe})$ meniscus lenses follow the lamp and are used to collimate and spatially filter the lamp output. ZnSe has low dispersion and absorption coefficients for our wavelength range. The diameter of the lenses is $12.7 \mathrm{~mm}$ and focal length $f=12.7 \mathrm{~mm}$, thus $f$-number $=1$. Referring to Fig. 2, the first lens is placed at focal length distance from the lamp filament so that the collected light is roughly collimated. The design clear aperture diameter of about $10 \mathrm{~mm}$ matches the beam output size to the input aperture of the AOTF. The light is then directed at 90 deg by a flat mirror to the spatial filter arrangement. The spatial filter consists of two ZnSe lenses approximately separated by the sum of their focal lengths with a 2-mm diameter aperture (pin hole) placed between the lenses. The spatial filter reduces background signal on the detector by eliminating off-axis rays that tend to scatter from surfaces in the AOTF housing and the PS fixture. During alignment, the spacing of the three lenses and aperture position was optimized in the laboratory to provide "best" collimation out of the spatial filter and to maximize the signal at the detector. Reflective optics were considered for this source collimation arrangement, but the lenses provided a more compact solution with little change in the collimation as a function of wavelength.

\subsection{Acousto-Optic Tunable Filter}

The collimated broadband light beam with a diameter of $10 \mathrm{~mm}$ (active lens size) exits the spatial filter and enters the AOTF, which has an effective input area of about $10 \times 10 \mathrm{~mm}$. The light is diffracted into two narrowband first-order beams $\left(o^{\prime}\right.$ and $\left.e^{\prime}\right)$ that separate at the output face of the AOTF angularly by $6.5 \mathrm{deg}$ from the zero-order broadband light. The PS only utilizes the $e^{\prime}$ beam, which passes through a slot that is machined through the back of an offaxis parabolic (OAP) mirror that follows the AOTF. Light from the $o^{\prime}$ beam is blocked by the mirror backside. The zero-order light ( $o$ and $e$ beams) is aligned with the input axis, and is mostly removed by a combination of baffling and antireflective paint coating the inner wall of the fixture. The remaining zero-order light is imaged at a point displaced from the detector.

For our application, the single diffracted order $\left(e^{\prime}\right)$ provides adequate performance and straightforward implementation. The single polarization efficiency for this firstorder beam was measured to be $68 \%$. So, for unpolarized input light, the overall efficiency of the channel is $\sim 34 \%$ at $3.39 \mu \mathrm{m}$ for $2 \mathrm{~W}$ RF power applied to the AOTF. Although it is possible to use both narrowband diffracted orders from the AOTF, that option was not considered since it requires the added complexity of a second detector channel. The AOTF for our PS is operated at an RF power of 2 to $3 \mathrm{~W}$, and is tuned at an RF range of 30.73 to $75.9 \mathrm{MHz}$ for the 1.6 to $3.6 \mu \mathrm{m}$ wavelength range.

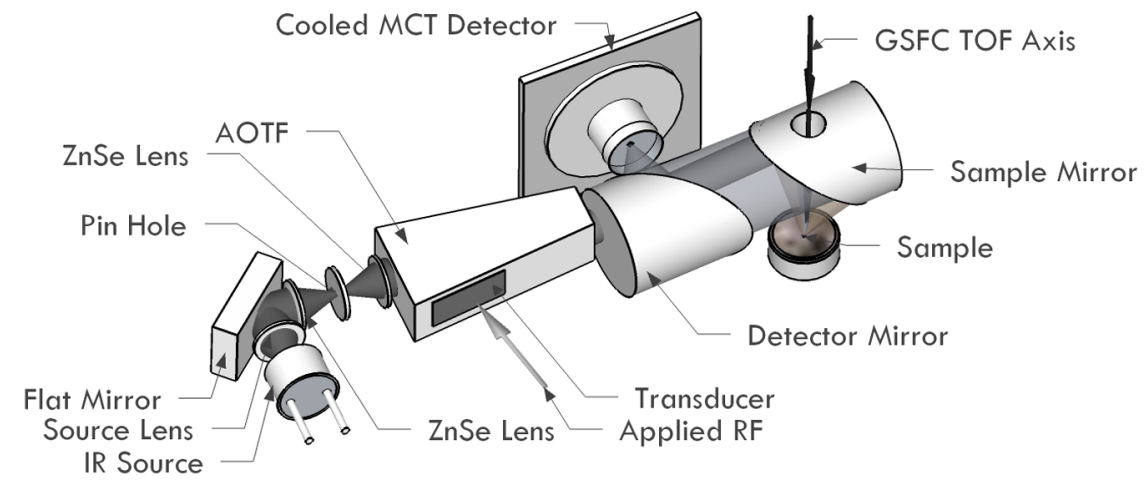

Fig. 2 A 3-D layout of the PS. 


\subsection{Off-Axis Parabolic Mirrors}

Following the AOTF, two matched OAP mirrors are arranged to focus the source beam onto the sample surface, collect the reflected light, and send it to the detector. The prescription for the two mirrors is the same: $25.4-\mathrm{mm}$ aperture, $f$-number $=1$, gold surface, and 90-deg reflection angle. Referring to Fig. 2, the $e^{\prime}$ beam passes from the AOTF through the slot in the first OAP (the "detector" mirror) and is incident on the second OAP (the "sample" mirror). The light is focused and directed at 90 deg (down) to the sample surface, which is $25.4 \mathrm{~mm}$ from the mirror vertex. On the sample surface, the arrangement forms a roughly 2-mm diameter spot that is a 2:1 magnification image of the lamp filament. Light scattered from the sample surface is collected by the sample OAP and then is redirected back to the detector OAP, which then sends it at a 90-deg angle to be focused on the detector. A spot size of approximately $2 \mathrm{~mm}$ is also formed at the detector although the active diameter of the detector is $1 \mathrm{~mm}$. The signal spot at the detector is oversized intentionally to provide some allowance for alignment.

A vertical hole of diameter $8 \mathrm{~mm}$ in the sample mirror (Fig. 2) is where the LDTOF-MS ion extraction lens can be inserted to draw laser-desorbed ions from the sample surface. Some obscuration by the ion lens is unavoidable since both instruments share the same field of view which results in a light efficiency loss of about $40 \%$.

\subsection{Fixture}

A monolithic fixture machined out of a single piece of aluminum holds the optical elements and detector. Figure 3 shows a design outline of the fixture with components (connectors were later relocated). An early photo of the optics assembly, prior to machining of the UV laser access port is shown in Fig. 4.

The OAPs are installed in a 25.4-mm diameter borehole where the sample OAP is fixed to a back plate on the side of the fixture and the detector OAP is held with set screws. The inner surface of the borehole was machined with large threads and was coated with IR antireflective paint to act as a grazing-incidence baffle to minimize scattered light. The

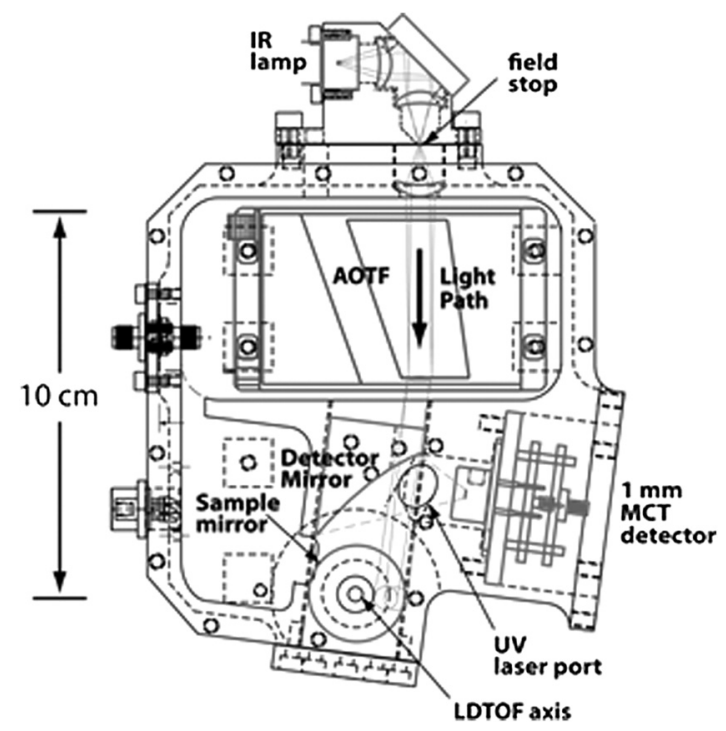

Fig. 3 Top view diagram of the PS fixture.

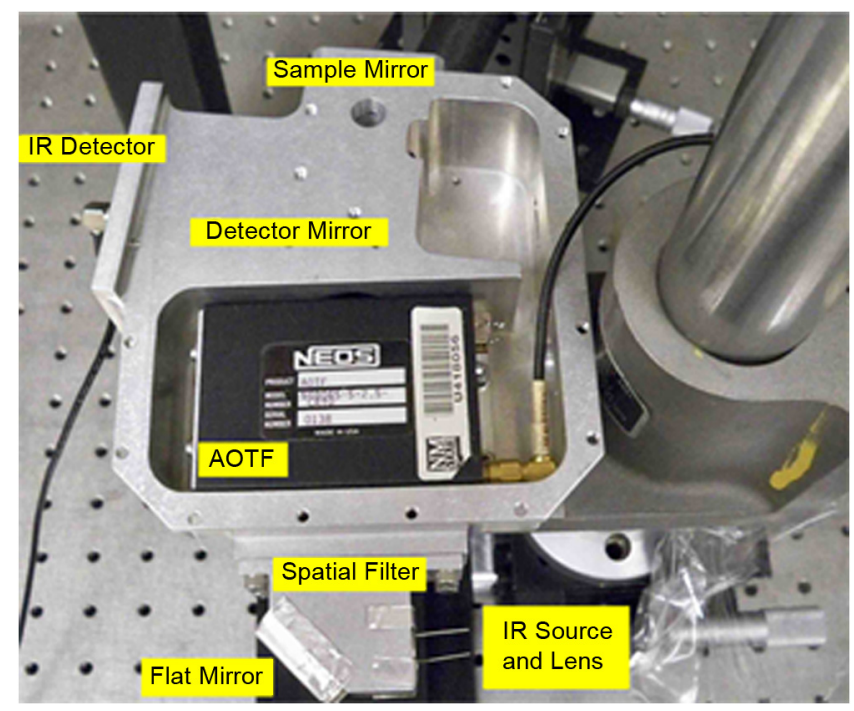

Fig. 4 A photo of the fixture (rotated by 180 deg compared to Fig. 3) showing the locations of the components.

OAP mirrors were adjusted to achieve a maximum output signal at the detector. The IR detector is inserted in a rectangular recessed area in the fixture and focus positioning was adjusted using shims. The sample is accessed through a hole in the bottom of the fixture where the sample surface is typically positioned about $5 \mathrm{~mm}$ from the bottom face of the fixture. Figure 4 shows the hole through the top of the fixture (near the sample mirror label) where the mass spectrometer's ion lens is inserted. The tip of the ion lens sits about $7 \mathrm{~mm}$ from the sample surface. Another hole was drilled later to one side to allow the mass spectrometer's UV laser to illuminate the sample surface.

\section{Electronics and Data Acquisition}

As indicated in the previous section, the PS is operated in a scanning mode where the AOTF bandpass is swept in time through the wavelength range of interest at a rate of several Hertz. Sweeping allows the detector output to be AC-coupled so that DC-signal offsets (e.g., optical scatter within the fixture) or a slowly changing thermal background can be removed before the signal is digitized. We found that a scan duration of about $100 \mathrm{~ms}$ is rapid enough to suppress ambient light fluctuations, but slow enough so that spectral features at the limit of instrument resolution will not be distorted by the finite signal bandwidth of the amplifier chain (Sec. 3.2). The electronics described in this section are used

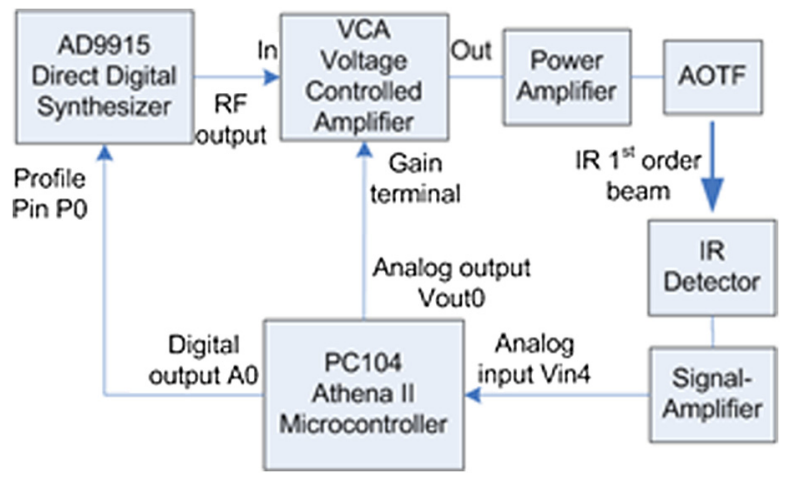

Fig. 5 Block diagram of the electronic components. 
Table 2 Electronic parts and specifications.

\begin{tabular}{lll}
\hline \hline $\begin{array}{l}\text { Device } \\
\text { DDS }\end{array}$ & \multicolumn{1}{c}{ Company } & \multicolumn{1}{c}{ Specifications } \\
PC104 & Diamolog Devices EVAL-AD9913 & Up to $100 \mathrm{MHz}$ analog output; 10-bit D/A converter; Output current 4.6 mA \\
VCA & Mini-Circuits ZFL-1000GH+ & Process speed $500 \mathrm{MHz}$; DDR DRAM $256 \mathrm{MB}$ \\
Power Amp & FD Comm. Corp. HD27334 & Freq. range 10 to $1200 \mathrm{MHz}$; Variable gain 0 to $24 \mathrm{~dB}$ \\
Detector & Teledyne Judson Technologies & Diameter $1 \mathrm{~mm}$; Mercury cadmium telluride (MCT); Wavelength range \\
& & 0.5 to $5.5 \mu \mathrm{m}$; Three-stage TE cooler; Transimpedance Gain $10^{5}$
\end{tabular}

to drive the AOTF, collect and digitize the signal provided by the detector, average multiple scans, and display and store the results. Figure 5 is a block diagram of the system and Table 2 lists the details of the components.

\subsection{Radio Frequency Chain}

The drive signal for the AOTF is generated by a chain of RF components. The initial RF waveform is produced by an AD9913 DDS chip. This DDS provides an output frequency up to $100 \mathrm{MHz}$, and a programmed ramp mode is utilized that generates linear sweeping frequency waveforms. Ramping starts when the DDS receives a trigger signal from the PC104 microcomputer. The synthesizer output, nominally about $0 \mathrm{dBm}$, is boosted by a voltage controller amplifier (VCA). The output of this stage is usually held constant at $22 \mathrm{~dB}$, but that can be changed by adjustment of a control voltage. The drive signal is applied to an RF power amplifier which has a fixed gain of $34 \mathrm{~dB}$ and provides the system with an extra constant gain to adequately drive the AOTF without exceeding a safe limit.

The power amplifier drives the AOTF with the desired RF power, which can be adjusted in software by an output setting in the DDS or by adjusting the gain on the VCA. Since the optical transmission efficiency of the AOTF varies with RF due to transducer impedance matching conditions, adjustable RF gain can be used to flatten the AOTF efficiency during a scan with predetermined gain adjustments.

\subsection{Detector Channel}

An "off-the-shelf" mercury cadmium telluride (MCT) detector with a wavelength sensitivity range of 0.5 to $5 \mu \mathrm{m}$ is used to detect the reflection signal. The AOTF has a design long wavelength transmission cutoff of about $3.4 \mu \mathrm{m}$, but provides usable response to about $3.5 \mu \mathrm{m}$. It is important for our application to extend the instrument response to the longest wavelength possible, thus the MCT detector was selected. Early tests of the spectrometer using an InAs detector showed high signal-to-noise, but with a shorter cutoff at about $3.2 \mu \mathrm{m}$. The MCT detector is cooled by a three-stage thermoelectric cooler (up to $-65^{\circ} \mathrm{C}$ below ambient) to reduce dark noise. The reflected light spot of about $2 \mathrm{~mm}$ in diameter overfills the detector diameter of $1 \mathrm{~mm}$, which effectively limits the sampling of the target surface to a spot of about $1 \mathrm{~mm}$ in diameter.
The output of the detector is sent to an AC-coupled signal amplifier. The amplifier consists of three stages where the first stage sets the $0.1 \mathrm{~Hz}$ cut-on frequency. The second stage is a Butterworth low-pass filter with a cut-off frequency of $5 \mathrm{kHz}$, which was chosen to be high enough that the filtering of the signal does not impact the fundamental spectral resolution of the instrument (set by the AOTF) during a scan. The third stage is a noninverting voltage amplifier with selectable gains ranging from 5 to 100 . The output signal of the amplifier is sent to the 16-bit ADC $(65,535$ samples, $76 \mu \mathrm{V}$ resolution) of the PC104 for data acquisition (DAQ) and processing.

\subsection{Data Collection and Control}

The PC104 is a single board computer that integrates an embedded PC and DAQ into a single board. One of the PC104's digital I/O lines is used to trigger the DDS frequency ramp. Since the DDS accepts only $1.8 \mathrm{~V}$, the $5 \mathrm{~V}$ pulse is sent through a voltage level shifter. A USB connection allows the PC104 to communicate the output power, frequency ramp range, and ramp time to the DDS. For synchronization purposes, the $\mathrm{I} / \mathrm{O}$ trigger pulse is also routed back to the external trigger of the PC104 DAQ to start the data collection. Thus the DDS and the DAQ are triggered independently, but at the same time, to scan and collect data simultaneously. Multiple scans (256 for the results shown in this paper) and their averages are obtained and stored on the PC104, where the signal is sampled at a rate of $10 \mathrm{kHz}$, which provides 1024 samples/scan. The software used to run the DDS and collect data is written in $\mathrm{C}++$.

\section{Spectral Resolution and Wavelength Calibration}

The transmission function for the AOTF used in the PS can be measured for a specific wavelength by removing the IR lamp source and sending the laser through the tuning optics along the optical axis. The injected light traverses the system and reflects off a gold-coated IR diffuse reflectance standard that acts as a high reflectivity Lambertian scattering surface so that the spectrometer records the transmission function at the laser wavelength as the AOTF scans. Measurements at both ends of the PS response range were made using an approximately $1.76 \mu \mathrm{m}$ wavelength laser diode beam and a $3.39 \mu \mathrm{m}$ HeNe laser beam. Figure 6 shows normalized transmission functions measured for these wavelengths. Measured FWHM values $(\Delta \lambda)$ were $0.004 \mu \mathrm{m}$ for $1.76 \mu \mathrm{m}$ 

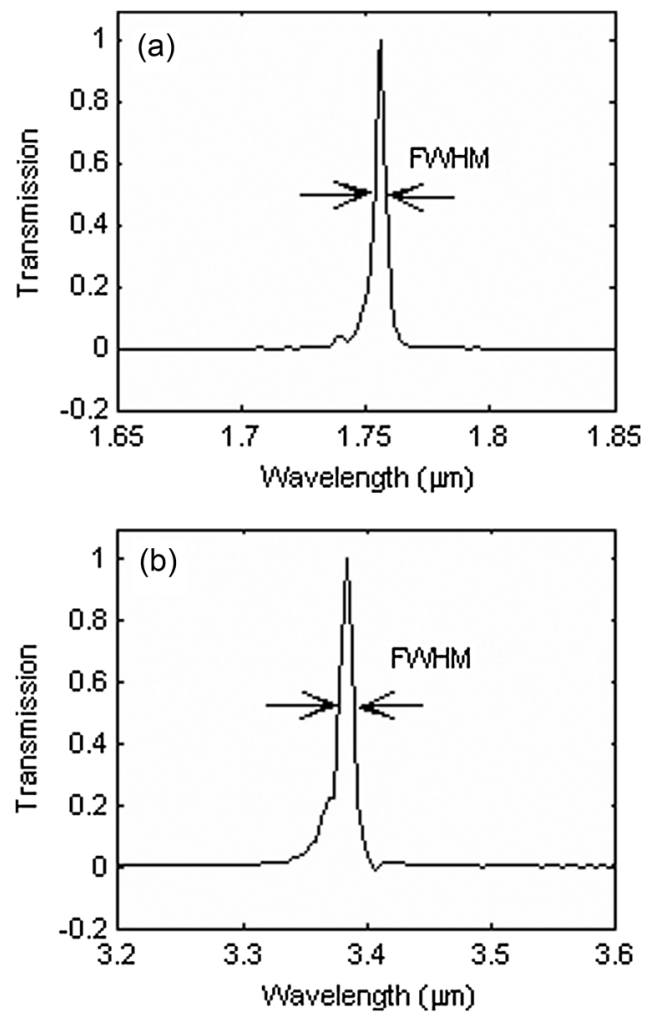

Fig. 6 Normalized transmission functions measured for (a) $1.76 \mu \mathrm{m}$ laser diode and (b) $3.39 \mu \mathrm{m}$ HeNe laser.

and $0.012 \mu \mathrm{m}$ for $3.39 \mu \mathrm{m}$, respectively. The spectral resolution $\lambda / \Delta \lambda$ is therefore 442.5 at $1.76 \mu \mathrm{m}$ and 282.5 at $3.39 \mu \mathrm{m}$, respectively.

The transmission function of an AOTF has been modeled in the literature as $T=T_{0} \sin \mathrm{c}^{2}\left[0.886\left(\lambda-\lambda_{0}\right) / \Delta \lambda\right]$, where $T_{0}$ is the peak transmission value at the center wavelength $\lambda_{0}{ }^{8}$ However, the measurements in Fig. 6 depart from the $\sin c^{2}$ form as they exhibit some asymmetry and broadening at the base with only minor indications of specific sidelobes. The broadening and sidelobes, which represent spectral leakage, tend to increase as the RF power applied to the AOTF increases. Although the peak transmission value also increases with the applied RF power, some optimization is required to maintain good transmission efficiency while limiting spectral leakage.

For an AOTF, the applied RF value and the center wavelength of the narrowband output are known to be inversely proportional. ${ }^{8}$ During a typical scan, data is recorded as a function of sample point where the RF step between points is constant. Therefore, an inverse relationship is found between the sample point and the narrowband wavelength. This relationship is known as the tuning curve and is critical to the spectral accuracy of the instrument.

The tuning curve for the PS was found in the laboratory using nine narrowband interference filters that span our wavelength range of interest. Each filter is placed at the input of the AOTF and a scan of the gold reflectance standard produces a narrow transmission feature whose sample peak corresponds to the interference filter's center wavelength. A data point at $3.39 \mu \mathrm{m}$ was also obtained using the IR HeNe laser. A polynomial function was fit to the data to yield the tuning relation (Fig. 7). Considering the wavelength range

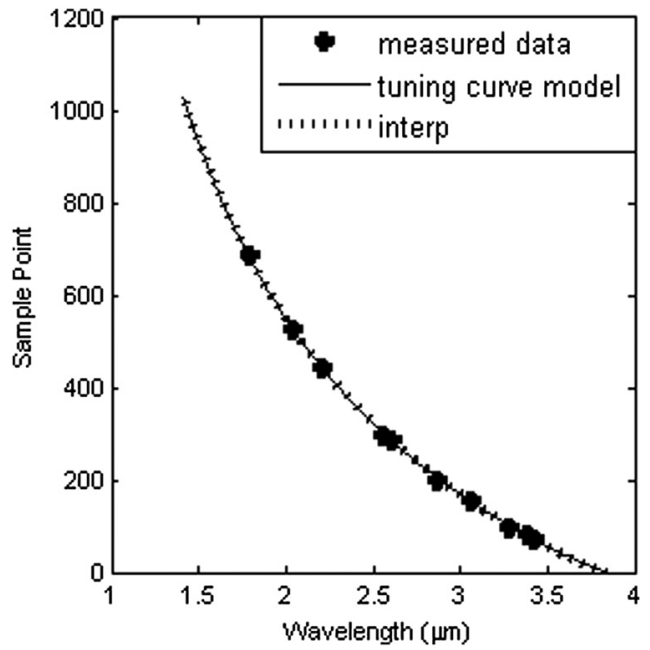

Fig. 7 Tuning curve model showing sample point versus wavelength: "measured data" indicates the filter and HeNe laser measurements, "tuning curve model" is a curve generated from Ref. 8 using the manufacturer specifications for the AOTF and "interp" indicates the polynomial fit.

and the number of sample points, we estimate the tuning curve accuracy is on the order of $1 \mathrm{~nm}$.

A "raw" (un-ratioed) signal output from the PS contains a variety of instrument and environment effects. Figure 8 shows a typical 256-scan average of the gold reflectance standard, which has a constant reflectivity throughout the wavelength range. The droop at each end of the curve is due to a decline in AOTF efficiency, the large amplitude periodic feature arises from RF mismatch between source and AOTF transducer, which produces standing waves in the cable, and is therefore a function of the RF cable length. The fine structure near $2.7 \mu \mathrm{m}$ is caused by water vapor in the measurement path. Fortunately, these artifacts are generally stable and are removed from a measurement by computing the ratio of a raw measurement scan to a raw reference standard scan.

\section{Radiometric Performance Model}

In this section, we examine the radiometric performance of the instrument through model calculations and comparisons with actual measurements. Three wavelengths that span our range of interest are considered. Parameters used in

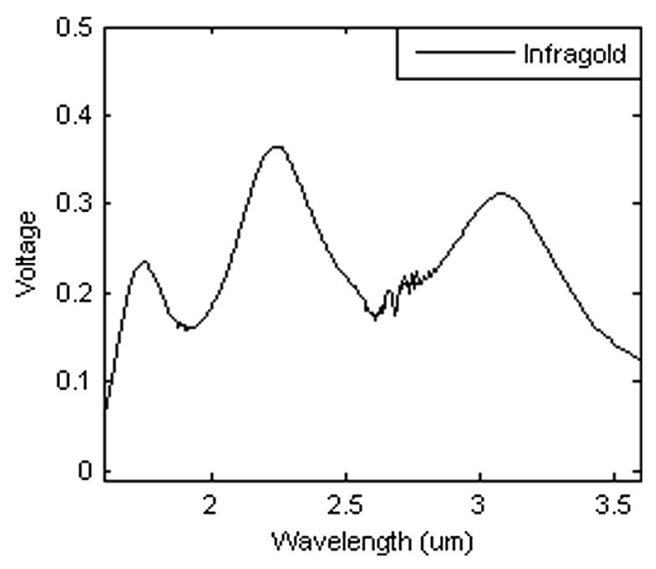

Fig. 8 Raw scan of the IR reflectance standard. 
Table 3 Transmission values.

\begin{tabular}{|c|c|c|c|c|}
\hline Component & Coating & Transmission & Number of surfaces & Total transmission \\
\hline Lamp Sapphire window & & 0.85 & 2 & 0.723 \\
\hline Flat mirror & Gold & 0.98 & 1 & 0.98 \\
\hline ZnSe lenses & $A / R$ & 0.975 & 6 & 0.859 \\
\hline AOTF & $A / R$ & 0.95 & 2 & 0.903 \\
\hline Sample reflectance $r_{s}$ & Gold & 0.95 & $(\times 1 / \pi)$ & 0.302 \\
\hline Sample mirror & Gold & 0.98 & 2 & 0.960 \\
\hline Detector mirror & Gold & 0.98 & 1 & 0.98 \\
\hline Total optics transmission $\left(T_{\mathrm{opt}}\right)$ & & & & 0.156 \\
\hline
\end{tabular}

Table 4 Source and system geometrical parameters.

\begin{tabular}{lc}
\hline \hline Parameter & \multicolumn{1}{c}{ Value } \\
\hline Source area $A_{s}$ & $1.2 \times 10^{-6} \mathrm{~m}^{2}$ \\
Spatial filter solid angle $\Omega_{\mathrm{SF}}$ & $0.366 \mathrm{sr}$ \\
Sample mirror solid angle $\Omega_{d}$ & $1.302 \mathrm{sr}$ \\
Sample mirror hole solid angle $\Omega_{d h}$ & $0.205 \mathrm{sr}$ \\
Detector's sensor area $A_{d}$ & $0.785 \times 10^{-6} \mathrm{~m}^{2}$ \\
Spot area on sample $A_{\text {spot }}$ & $4.8 \times 10^{-6} \mathrm{~m}^{2}$ \\
OAP mirror area $A_{\mathrm{OAP}}$ & $0.592 \times 10^{-3} \mathrm{~m}^{2}$ \\
Hole in detector OAP mirror area $A_{h 1}$ & $53.3 \times 10^{-6} \mathrm{~m}^{2}$ \\
\hline \hline
\end{tabular}

the calculations are shown in Tables 3 and 4. Component transmission values were taken from the manufacturers' specifications or from typical values in the literature. The assumed sample is the gold IR reflectance standard.

Table 5 presents the combined system parameters and the predicted flux at the detector. The lamp spectral radiance $L_{e, \lambda}\left(\mathrm{W} \mathrm{m}^{-2} \mu \mathrm{m}^{-1} \mathrm{sr}^{-1}\right)$ is assumed to be distributed like a blackbody source

$L_{e, \lambda}=\frac{\epsilon C_{1}}{\pi \lambda^{5}\left[\exp \left(C_{2} / \lambda T\right)-1\right]}$, where $T=1900 \mathrm{~K}, C_{1}=3.7418 \times 10^{4}\left(\mathrm{~W}-\mu \mathrm{m}^{4}-\mathrm{cm}^{-2}\right)$, $C_{2}=1.43877 \times 10^{4}(\mu \mathrm{m}-\mathrm{K})$ and $\lambda$ is the wavelength in $\mu \mathrm{m}$. The emissivity of the lamp, $\epsilon$, ranges from $\sim 0.1$ to 0.2 at the color temperature of our lamp filament $(1900 \mathrm{~K}) .{ }^{17}$ For purposes of estimating performance, we used 0.17 . The FWHM of the AOTF transmission function $\Delta \lambda$ was measured in the laboratory for the 1.76 and $3.39 \mu \mathrm{m}$ wavelengths (see Sec. 4). $\Delta \lambda$ for the $2.6 \mu \mathrm{m}$ wavelength was interpolated from the other two. The AOTF transmission efficiency $T_{\text {AOTF }}$ is a rough estimate based on the laboratory measurements. In practice, the efficiency can vary depending on the wavelength, the applied RF power, and the configuration of the RF cabling (impedance matching with the AOTF transducer). The flux at the detector is calculated using

$$
\phi=L_{e, \lambda} \Delta \lambda A_{s} \Omega_{\mathrm{SF}} T_{\mathrm{AOTF}} T_{\mathrm{opt}}\left(\Omega_{d}-\Omega_{d h}\right) \frac{A_{d}}{A_{\mathrm{spot}}} \frac{A_{\mathrm{OAP}}-A_{h 1}}{A_{\mathrm{OAP}}} .
$$

The voltage at the output of the signal amplifier is related to the detector flux $\phi$ by $V_{\text {calc }}=R \phi G$, where $R$ is the detector responsivity and $G=10^{6}$ is the product of detector transimpedance gain $\left(10^{5}\right)$ and signal-amplifier gain (10). Table 6 shows $V_{\text {calc }}$ along with an example of the measured voltage values $V_{\text {meas }}$ (which is subject to change) obtained with the PS in the laboratory. The measured value was obtained by observing the gold IR reflectance standard and averaging 256 individual scans. We found that the 256-scan average is a good compromise between noise reduction and data collection speed. The calculated and measured voltages are generally consistent although the measured values are

Table 5 Calculated optical flux at the detector for three selected wavelengths.

\begin{tabular}{|c|c|c|c|c|c|c|c|c|c|c|}
\hline$\lambda(\mu \mathrm{m})$ & $L_{e, \lambda}\left(\mathrm{W} / \mathrm{m}^{2}-\mathrm{sr}\right)$ & $\Delta \lambda(\mu \mathrm{m})$ & $A_{s} \mathrm{~mm}^{2}$ & $\Omega_{\mathrm{SF}}(\mathrm{sr})$ & $T_{\text {AOTF }}$ efficiency & $T_{\mathrm{opt}}$ & $\Omega_{d}-\Omega_{d h}(\mathrm{sr})$ & $\frac{A_{d}}{A_{\text {spot }}}$ & $\frac{A_{O A P}-A_{h 1}}{A_{O A P}}$ & $\phi(\mathrm{nW})$ \\
\hline 1.76 & $16.45 \times 10^{3}$ & 0.004 & 1.2 & 0.366 & 0.3 & 0.1562 & 1.1 & 0.164 & 0.91 & 221 \\
\hline 2.6 & $9.79 \times 10^{3}$ & 0.0075 & 1.2 & 0.366 & 0.3 & 0.1562 & 1.1 & 0.164 & 0.91 & 247 \\
\hline 3.39 & $5.43 \times 10^{3}$ & 0.012 & 1.2 & 0.366 & 0.3 & 0.1562 & 1.1 & 0.164 & 0.91 & 219 \\
\hline
\end{tabular}


Table 6 Comparison between measured and calculated voltage at the detector.

\begin{tabular}{lcccccc}
$\lambda(\mu \mathrm{m})$ & $\phi(\mathrm{nW})$ & $R(\mathrm{~A} / \mathrm{W})$ & $V_{\text {calc }}(\mathrm{mV})$ & $V_{\text {meas }}(\mathrm{mV})$ & $\sigma(\mathrm{mV})^{\mathrm{a}}$ & $\mathrm{NER}(\%)^{\mathrm{a}}$ \\
\hline 1.76 & 221 & 0.63 & 139 & 233 & 0.25 & 0.104 \\
2.6 & 247 & 1.13 & 279 & 181 & 0.28 & 0.154 \\
3.39 & 219 & 1.55 & 339 & 180 & 0.26 & 0.144 \\
\hline \hline
\end{tabular}

a256-scan average.

lower for the longer wavelengths. Some differences are expected as the variability with wavelength of the source emissivity and AOTF efficiency was not considered. The standard deviation $\sigma$ reported in Table 6 was obtained in the laboratory using multiple measurements and corresponds to a 256-scan average. The last entry in Table 6 is a "noise equivalent reflectance" value given by NER = $\sigma / V_{\text {meas }} \times 100 \%$. These results indicate that at high signal levels, e.g., when observing the gold reflectance standard, the noise level for the 256-scan average is equivalent to a reflectance change of about $0.13 \%$.

\section{Example Spectra}

The PS has been applied in the laboratory to a variety of mmsized mineral samples including sulfates, carbonates, iron oxides, and clay powder. In this section, we compare a few examples of measured spectra with reference spectra from
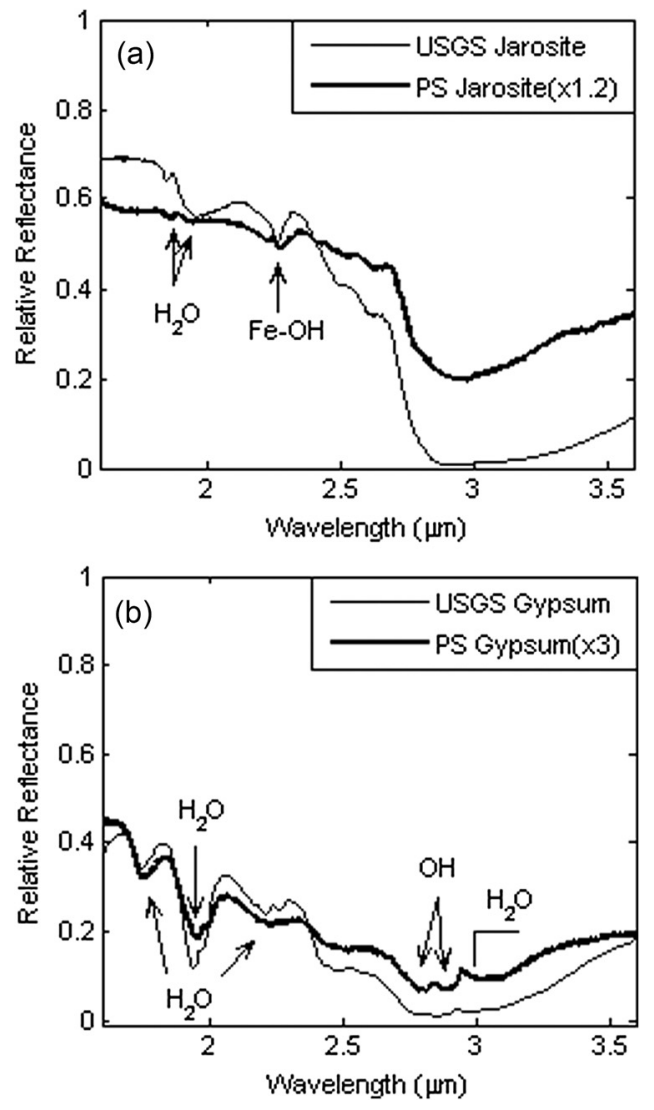

Fig. 9 Sample spectra of the sulfates (a) jarosite and (b) gypsum compared to the USGS data.
United States Geological Survey (USGS) data. ${ }^{18}$ All the PS results are 256-scan averages. In general, the average sample reflectance as measured by the PS can differ appreciably from the USGS data. This is likely a result of grain size differences, degree of sample hydration, and differences in spectral resolution between the AOTF and reference spectra. Another factor may be that the PS sample illumination is linearly polarized whereas other measurements utilize unpolarized light. Except for the clays, our samples were small chips and the average measured reflectance changed considerably with positioning of the sample. It is notable that the PS average reflectance values for the clay powders were very similar to the USGS data. To better compare relative spectral features in the other cases, the PS curve values have been multiplied by a factor which is indicated in the figure legend. The lower reflectance in the PS measurements may be due to sample positioning, referring our spectra to an infragold standard, room temperature, testing location, and sample orientation. Overall, most of the salient features such as chemical bonding absorption minima exhibited by the USGS spectra seem to be captured by the PS measurements.

Figure 9(a) shows the spectral reflectance of the sulfate jarosite. The curve exhibits water features close to 1.8 and $1.9 \mu \mathrm{m}$ and iron oxide (Fe-OH) features around $2.2 \mu \mathrm{m}$. The result for gypsum [Fig. 9(b)], another sulfate, exhibits water features at $1.75,1.95,2.23,2.28 \mu \mathrm{m}$ and beyond $2.97 \mu \mathrm{m}$. Hydroxide features are present between 2.8 and $2.9 \mu \mathrm{m}$.

The spectral signatures of two carbonates, calcite and dolomite, are shown in Fig. 10. For calcite, carbonate ion
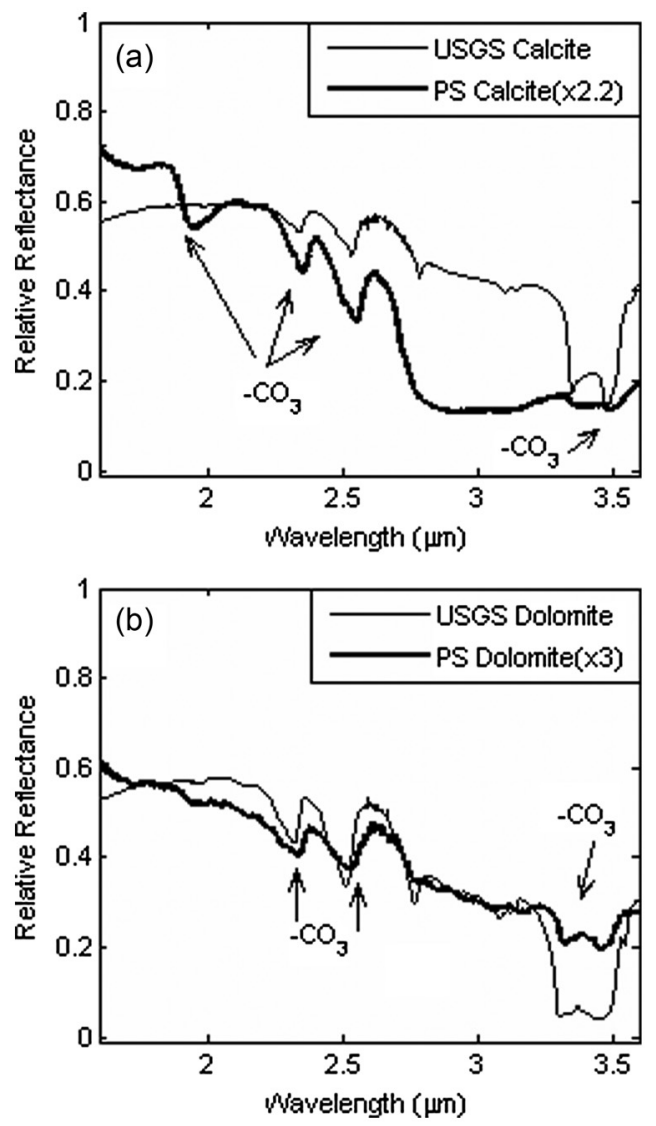

Fig. 10 Sample spectra of the carbonates (a) calcite and (b) dolomite compared to the USGS data. 

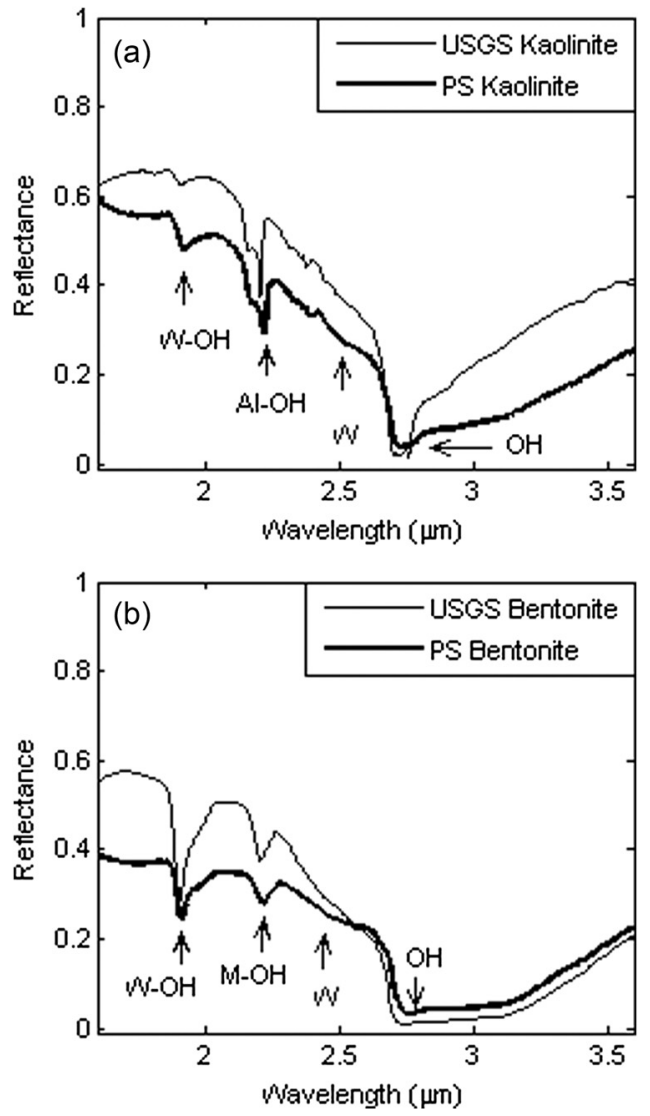

Fig. 11 Sample spectra of the clays (a) kaolinite and (b) bentonite compared to the USGS data.

features are evident at wavelengths around 1.9, 2.35, and $2.55 \mu \mathrm{m}$. Similar features are seen for dolomite, although the strong hydration dip at wavelengths longer than about $2.7 \mu \mathrm{m}$ is not as significant in the dolomite spectrum.

Spectra for the clays, kaolinite, and bentonite, are shown in Fig. 11. In both samples, the combination of water-bend and $\mathrm{OH}$-stretch is present at $1.9 \mu \mathrm{m}$. This combination requires the presence of both water and hydroxide. At $2.22 \mu \mathrm{m}$, a metal-OH combination is observed, for kaolinite the feature is $\mathrm{Al}-\mathrm{OH}$ and for bentonite it is $\mathrm{M}-\mathrm{OH}$. Water and hydroxide features are shown around wavelengths 2.56 and $2.73 \mu \mathrm{m}$, respectively.

For iron oxides, goethite and hematite were examined. Figure 12 shows that hematite has no clear features except for water absorption shown around $3 \mu \mathrm{m}$, but for goethite, absorption is present at 1.9 and $2.4 \mu \mathrm{m}$.

\section{Conclusions}

A compact IR reflectance PS based on AOTF technology has been constructed with a wavelength range of 1.6 to $3.6 \mu \mathrm{m}$. The instrument provides in situ detection for organics or organically modified minerals via their spectral signature at a scale size of a millimeter on a sample surface at a distance of about $5 \mathrm{~mm}$. Design features include: (1) a common sample point accessible to both the PS and a companion LDTOF-MS instrument, (2) a shared set of OAP mirrors for illumination and reflectance signal collection, (3) detection with a single TE-cooled MCT photodiode, and (4) an AC-coupled measurement approach to remove background signal. Radiometric measurements were close to expected
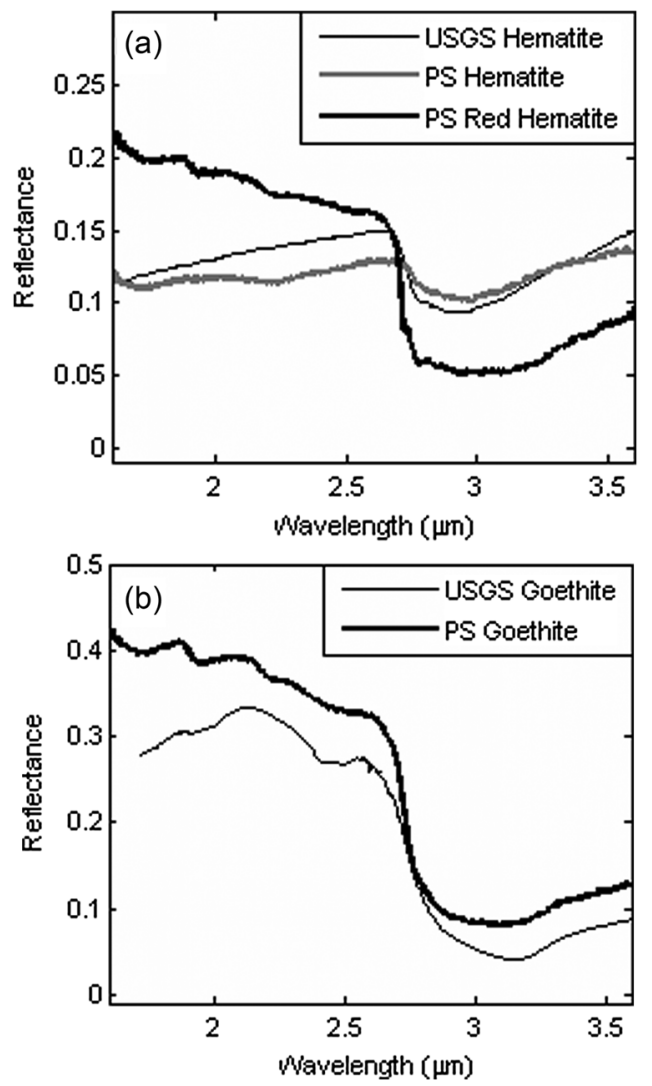

Fig. 12 Sample spectra of the iron oxides (a) hematite and (b) goethite compared to the USGS data.

values. The noise equivalent reflectance indicates that at high signal levels, for a 256-scan average the noise level is equivalent to a reflectance change of about $0.13 \%$. The measured spectral resolution was found to be around 280 for $3.39 \mu \mathrm{m}$ and 440 for $1.76 \mu \mathrm{m}$, respectively. The reflectance signature obtained for eight minerals were presented and show good correspondence to USGS data.

The PS is intended to be integrated with the LDTOF-MS to examine and analyze samples collected in situ from planetary surfaces to search for organic biomarkers or mineralogical signatures that imply the existence or lack of organic materials, and it can also perform as a standalone device for field measurements. Future work will include the development and examination of standardized targets with known IR spectral absorption characteristics as well as detailed investigations of the spectral features for additional minerals and organic constituents.

\section{Acknowledgments}

This project was sponsored by NASA through the Astrobiology Science and Technology Instrument Development (ASTID) program and the Experimental Program to Stimulate Competitive Research (EPSCoR) program. Also special thanks to Kyle Uckert for assisting with data collection and processing, and Vinush Depala for data acquisition system development and testing.

\section{References}

1. N. J. Chanover et al., "An AOTF-LDTOF spectrometer suite for in situ organic detection and characterization," in IEEE Aerospace Conference, pp. 1-13, IEEE, Piscataway, NJ (2011). 
2. E. S. Wachman, W. Niu, and D. L. Farkas, "AOTF microscope for imaging with increased speed and spectral versatility," Biophys. J. 73(3), 1215-1222 (1997).

3. B. Rajwa et al., "AOTF-based system for image cytometry," Proc. SPIE 5694, 16-23 (2005)

4. D. A. Glenar, D. L. Blaney, and J. J. Hillman, "AIMS: acousto-optic imaging spectrometer for spectral mapping of solid surfaces," Acto Astronaut. 52(2-6), 389-396 (2003).

5. J.-L. Bertaux et al., "SPICAV on Venus Express: Three spectrometers to study the global structure and composition of the Venus atmosphere," Planet. Space Sci. 55(12), 1673-1700 (2007).

6. O. Korablev et al., "An AOTF-based spectrometer for the studies of Mars atmosphere for Mars Express ESA mission," Adv. Space Res. 29(2), 143-150 (2002).

7. O. Korablev et al., "An AOTF-based spectrometer for Mars atmosphere sounding," Proc. SPIE 4818, 261-271 (2002)

8. D. Glenar et al., "Acousto-optic imaging spectropolarimetry for remote sensing," Appl. Opt. 33(31), 7412-7424 (1994).

9. G. Georgiev, D. A. Glenar, and J. J. Hillman, "Spectral characterization of acousto-optic filters used in imaging spectroscopy," Appl. Opt. 41(1), 209-217 (2002)

10. N. J. Chanover et al., "Probing Titan's lower atmosphere with acoustooptic tuning," Icarus 163(1), 150-163 (2003).

11. D. A. Glenar et al., "Multispectral imagery of Jupiter and Saturn using adaptive optics and acousto-optic tuning," Publ. Astron. Soc. Pac. 109, 326-337 (1997).

12. J. Vila-Francés et al., "Configurable-bandwidth imaging spectrometer based on an acousto-optic tunable filter," Rev. Sci. Instrum. 77(073108), $1-10(2006)$

13. N. Gupta, R. Dahmani, and S. Choy, "Acousto-optic tunable filter based visible- to near-infrared spectropolarimetric imager," Photo-Opt. Instrum. Eng. 41(5), 1033-1038 (2002)

14. F. B. Gonzaga and C. Pasquini, "Near-infrared emission spectrometry based on an acousto-optical tunable filter," Anal. Chem. 77(4), 1046-1054 (2005).

15. N. J. Chanover, D. A. Glenar, and J. J. Hillman, "Multispectral near-IR imaging of Venus nightside cloud features," J. Geophys. Res. 103(E13), 31335-31348 (1998).

16. N. J. Chanover et al., "Rapid assessment of high value samples: an AOTF-LDTOF spectrometer suite for planetary surfaces," in IEEE Aerospace Conference, pp. 1-10, IEEE, Piscataway, NJ (2012).

17. V. D. Dmitriev and G. K. Kholopov, "Radiant emissivity of tungsten in the infrared region of the spectrum," J. Appl. Spectros. 2(6), 315-320 (1965).

18. R. N. Clark et al., "USGS Digital Spectral Library splibs06a, U.S Geological Survey, Data Series 231," 2007, http://speclab.cr.usgs .gov/spectral.lib06/ds231/datatable.html (July 2012).

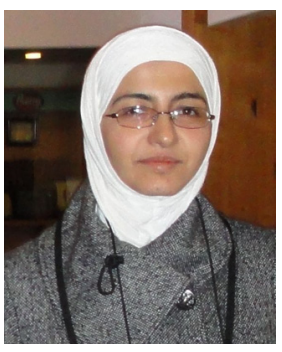

Rula Tawalbeh is a doctoral student in the Klipsch School of Electrical and Computer Engineering at New Mexico State University. She graduated from Yarmouk University, Jordan, in 1997 and earned the MSc degree from Jordan University of Science and Technology in 2001. She is interested in free-space optics, spectroscopy, and optical networks.

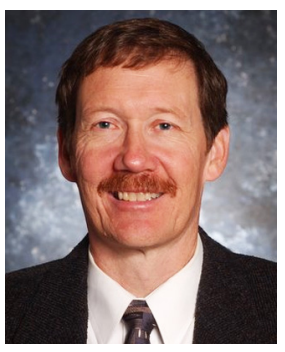

David Voelz is a professor in the Klipsch School of Electrical and Computer Engineering at New Mexico State University. He has been involved in the development of optical systems involving AOTF components since 2002. His research interests include spectral and polarization sensing, laser beam propagation through turbulence, laser communications, and imaging theory. He earned a PhD EE degree from the University of Illinois in 1987.

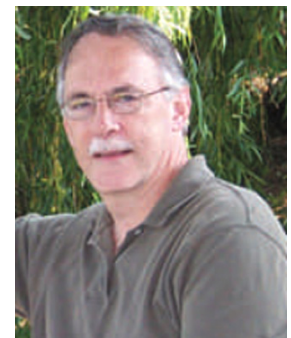

David Glenar is an emeritus planetary scientist at NASA's Goddard Space Flight Center, where his research interests included high resolution spectroscopy, applications of AOTFs for spectral imaging, and radiative transfer modeling. $\mathrm{He}$ is currently senior research associate in the Astronomy Department at New Mexico State University and is also a member of the National Lunar Science Institute.

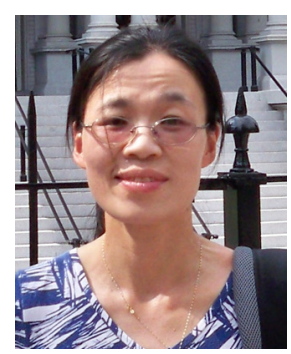

Xifeng Xiao received her BS and MS degrees in physics from Xiamen University Fujian, China, in 1998 and 2001, respectively. She earned her MS and PhD degrees in electrical engineering from New Mexico State University in 2004 and 2008, respectively, where she is currently a research assistant professor. Her research interests include simulation and modeling of freespace laser communication, liquid-crystal polarization, and demonstration and implementation of AOTF technologies.

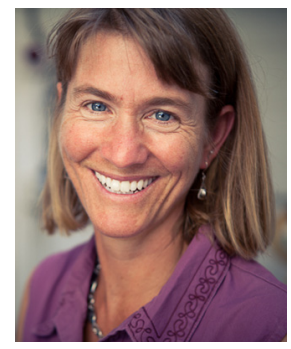

Nancy Chanover is an associate professor of astronomy at New Mexico State University and serves as the principal investigator of the project described in this paper. She has been involved in the demonstration and implementation of AOTF technologies for planetary science since 1997. Her research interests include the vertical structure and dynamics of planetary atmospheres and instrument development. She earned her PhD from New Mexico State University in 1997.

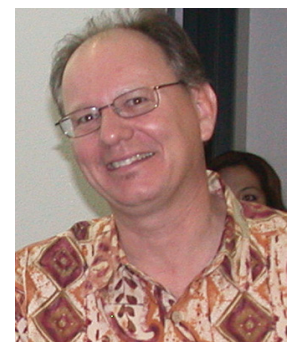

Robert Hull is a senior electronics technician with the Klipsch School. His background includes RF, telemetry and integration experience with sounding rockets, missile systems and Space Shuttle payloads. He came to NMSU to support high-altitude balloon research in cosmic ray physics. He is currently the EE capstone coordinator for senior team projects and the technical coordinator for the BEST robotics outreach program. For the Optics Laboratory, Robert provides design, fabrication and test support for complex optical and electronic configurations.

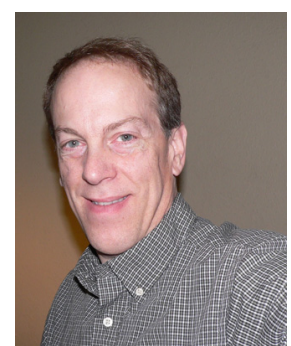

David Kuehn is a professor in the Physics Department at Pittsburg State University, Pittsburg, Kansas. He has been involved with the development of infrared and optical AOTF imaging systems since 2000. His research interests include the vertical structure, composition, and dynamics of outer planet atmospheres. He earned his $\mathrm{PhD}$ in astronomy from New Mexico State University in 1990. 